\title{
Transseptal puncture without fluoroscopy using a radiofrequency needle: A case series
}

\author{
Guram Imnadze ${ }^{1,2}$, Tarek Ajaj ${ }^{1}$, Hendrik Bante ${ }^{1}$, Christian Sohns ${ }^{2}$, Philipp Sommer ${ }^{2}$ \\ ${ }^{1}$ Arrhythmia Department, Klinikum Osnabrueck, Germany \\ ${ }^{2}$ Clinic for Electrophysiology, Herz- und Diabeteszentrum NRW, \\ Ruhr-Universität Bochum, Bad Oeynhausen, Germany
}

\begin{abstract}
Background: The non-fluoroscopy approach with the use of a three-dimensional (3D) navigation system is increasingly recognized as a future technology in the treatment of arrhythmias. However, there are a limited number of articles published concerning transseptal puncture without the use of fluoroscopy. Methods: Presented in this paper is the first series of patients $(n=10)$ that have undergone transseptal puncture without the use of fluoroscopy under transesophageal echocardiography control using a radiofrequency transseptal needle and a $3 D$ navigation system.

Results: All patients were treated without complications. In 6 patients, re-pulmonary vein isolation was performed. In 5 cases, linear ablation of the left atrium for treatment of left atrial macro re-entry tachycardia was provided. In 2 patients, focal atrial tachycardia was treated, 1 patient underwent cavo tricuspidal isthmus (CTI) ablation and 1 patient, re-CTI ablation. The ablation of complex fragmented atrial electrograms was done in 2 patients. In 1 case, right atrial macro re-entry tachycardia was treated. Conclusions: Transseptal puncture without using fluoroscopy is safe and effective when using a radiofrequency needle, a 3D navigation system and transesophageal echocardiography. (Cardiol J 2021; 28, 5: 655-662)
\end{abstract}

Key words: non-fluoroscopic approach, transseptal puncture, radiofrequency needle, three-dimensional mapping system

\section{Introduction}

Interest in non-fluoroscopy radiofrequency (RF) ablation (RFA) for the treatment of supraventricular tachycardia (SVT) arising from the right atrium (RA) has increased greatly over the last decade [1]. A new generation of three-dimensional (3D)-navigation systems allow the operator to build a map of the right-sided chambers with no, or minimal use of fluoroscopy. Number of publications in accordance with the safety and efficacy of non-fluoroscopy RFA of SVT have recently been published [2, 3]. Little is known about the safety and efficacy of transseptal puncture (TSP) using the non-fluoroscopy technique. As such, this paper presents a series of TSPs and RFA of the left atrium
(LA) using a RF needle, 3D navigation system and transesophageal echocardiography (TEE), without the use of fluoroscopy.

\section{Methods}

Thirteen consecutive patients were enrolled in this study. Three patients were excluded because of persistent foramen ovale or interatrial septal (IAS) defect after the first RFA. Nevertheless, they were also successfully ablated using the no-fluoroscopy technique.

In 10 patients ( 6 female) with a mean age of $70.6 \pm 6.5$ years, an RF needle was used for TSP. All patients underwent initial ablation therapy for atrial fibrillation (AF). Nine patients had pulmonary

Address for correspondence: Guram Imnadze, MD, Head of Arrhythmia Department, Klinikum Osnabrück, Am Finkenhügel 1, Osnabrück, Germany 49076, tel: +49 173 3738489, e-mail: imnadze@web.de

Received: 10.07.2019 Accepted: 24.12.2019 Early publication date: 21.01.2020

This article is available in open access under Creative Common Attribution-Non-Commercial-No Derivatives 4.0 International (CC BY-NC-ND 4.0) license, allowing to download articles and share them with others as long as they credit the authors and the publisher, but without permission to change them in any way or use them commercially. 
vein isolation (PVI) using cryoballoon, 1 patient had PVI using an RF catheter and 2 patients had re-PVI using an RF catheter after the first PVI procedure was conducted using a cryoballoon. Two patients had paroxysmal form and 8 patients had a persistent form of AF. Typical atrial flutter was documented in 4 cases. Eight patients had arterial hypertension, 2 patients suffered from apoplexy, 1 patient had diabetes, and 1 patient had chronic obstructive pulmonary disease (COPD). In 2 patients, ischemic heart disease was diagnosed, one was treated with coronary artery bypass grafting and the other with coronary stenting. None of the patients had implanted cardiac devices.

All procedures were done under sedation and uninterrupted anticoagulation. Intravenous infusion of Propofol and Fentanyl injection was used. Intravenous heparin was administered during the procedure to maintain an activated clotting time (ACT) in excess of $300 \mathrm{~s}$.

Vascular access was obtained from the right femoral vein. $4 \mathrm{~F}$ and $8 \mathrm{~F}$ short sheaths and one $8.5 \mathrm{~F}$ long sheath (SL1 with atraumatic tip, SWARTZ, Abbott, USA) was inserted (see details below).

The 3D navigation system (Ensite Precision ${ }^{\mathrm{TM}}$ Cardiac Mapping System, Abbott, USA) was used in all cases. Initial anatomical mapping of right-sided heart structures was performed using a $4 \mathrm{~F}$ decapolar steerable diagnostic catheter (Inquiry ${ }^{\mathrm{TM}}$, Abbott, USA), which then was placed in the coronary sinus (CS). For mapping of the LA and the pulmonary veins, a circular mapping catheter (Advisor ${ }^{\mathrm{TM}} \mathrm{FL}$, Sensor Enabled ${ }^{\mathrm{TM}}$, Abbott, USA) or a multipolar mapping catheter (Advisor ${ }^{\mathrm{TM}}$ HD Grid, Sensor Enabled $^{\mathrm{TM}}$, Abbott, USA) was used. Mapping and ablation were performed with an irrigated ablation catheter (Flexability ${ }^{\mathrm{TM}}$, Abbott, USA). The TSP was performed with a RF-Needle $\left(\mathrm{NRG}^{\circledR}\right.$ Transseptal Needle, Baylis Medical Company Inc., Canada) in all cases. All TSPs were performed under TEE imaging using a compact cardiovascular ultrasound system (CX50, PHILIPS, Netherland) with a TEE array transducer (X7-2t, PHILIPS, Netherlands).

\section{The TSP with RF needle}

The technique was divided into three steps.

1. Right-sided anatomical mapping. The anatomical mapping of right-sided structures using a steerable diagnostic catheter is safe and effective, and does not take a lot of time. First, mapping of the venous system and the inferior vena cava (IVC) was undertaken. Followed by mapping of the RA especially the IAS, superior vena cava (SVC) and the CS. Afterwards, a long transseptal sheath was inserted into the femoral vein over a long wire $(10-20 \mathrm{~cm})$ to ensure that the tip of the sheath was in the venous system. After the dilator and wire was withdrawn, the sheath was aspirated and flushed with saline and the ablation catheter was inserted. Once the RF catheter was in the venous system, its trajectory was tracked through the 3D anatomical course of the venous system which was established using a diagnostic catheter. Once the ablation catheter was placed in the SVC, the long sheath was slid carefully over it. It was inserted until an impedance difference occurred (once the tip of the sheath made contact with the catheter electrodes) and the catheter shape was moved out from the 3D anatomical map borders. This confirmed "housing" of the RF catheter in the sheath. Then the catheter was withdrawn and the sheath was aspirated and flushed again with saline (Fig. 1, Suppl. Video 1).

2. The blind phase. We called this phase blind because it could not be completely controlled with the $3 \mathrm{D}$ navigation system and the TEE. Once the SL1 sheath was placed in the desired position, it was fixed and the dilator was inserted over the wire. To avoid uncontrolled contact of the dilator with the SVC tissue, it was inserted into the sheath until marker " 2 " on the dilator. After the wire was withdrawn the dilator was aspirated and flushed with saline.

The RF-needle was then inserted into the dilator in consideration of special markers. The $\mathrm{RF}$ needle has a short pin directly after the handle part, from this pin the needle arises. The distance from the end of this pin to the dilator orifice should be equal to the length of the pin itself (Fig. 2A). The RF needle was then flushed with saline and connected to the cable and pressure line. Once this position was reached, the RF-needle together with the dilator was fixed and the sheath was slid gently back until a connection (click) with the dilator occurred (Fig. 2B). Afterwards, the sheath and the dilator were sliding together backwards for $2-3 \mathrm{~mm}$, while the RF needle remained fixed. Once the tip of the RF needle left the dilator, a unipolar point appeared on the 3D map (Fig. 2C). The whole assembly of the sheath, dilator and the needle were now slid back until a drop into the fossa ovalis occurred. It should be noted that the needle and the sheath position were similar to the standard TSP technique.

3. Transeptal puncture. Once the RF-needle was pulled back from the SVC and dropped into the fossa ovalis, the TEE image showed the "tenting" of the interatrial septum, ensuring a correct needle 


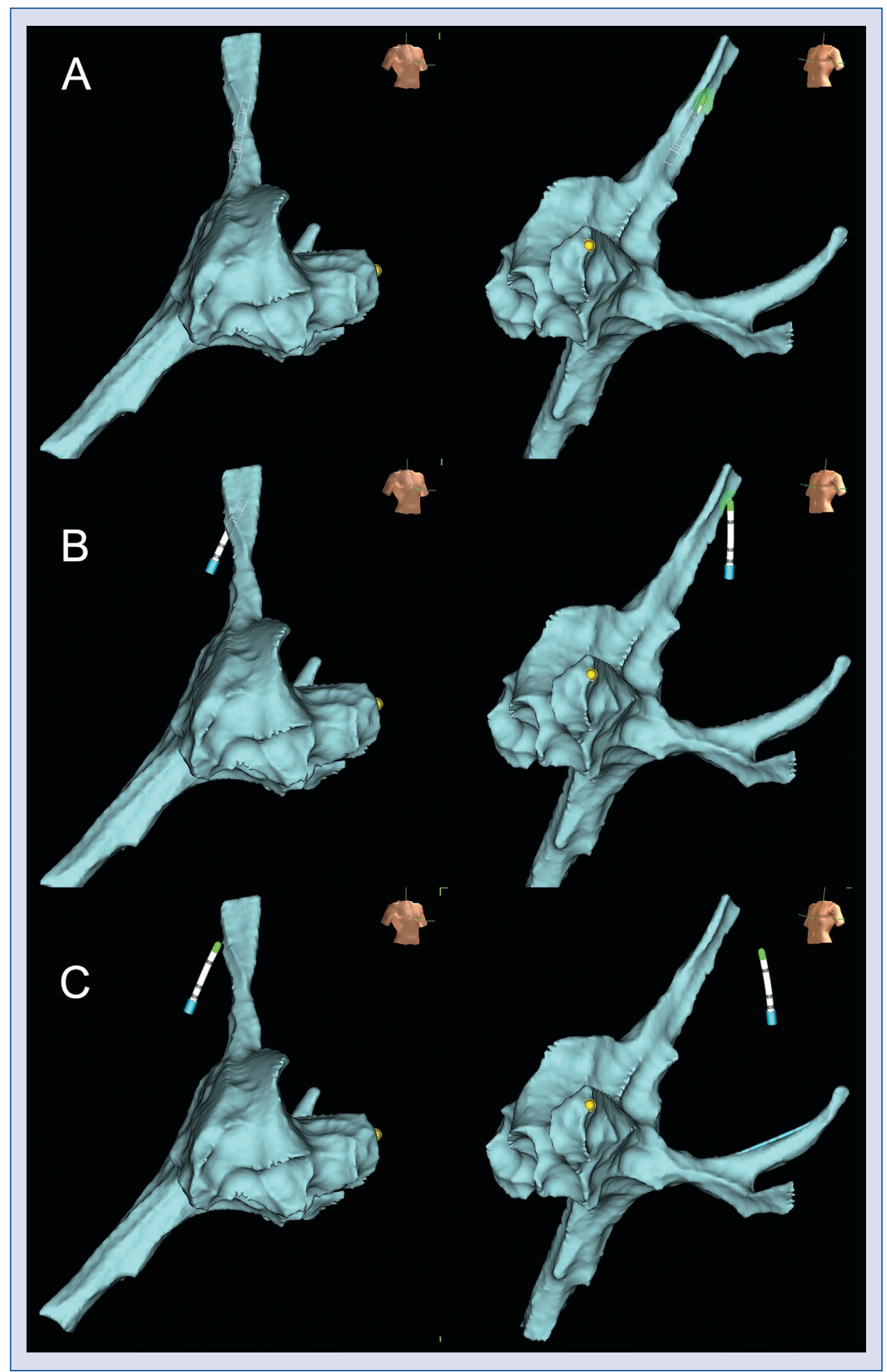

Figure 1. An anatomical map of the right atrium, inferior and superior vena cava and coronary sinus. The bundle of His is marked with a yellow point. The multipolar diagnostic catheter placed in the coronary sinus (blue color). On the left side, the right anterior oblique projection is presented. On the right side, the left anterior oblique projection is presented; $\mathbf{A}$. The ablation catheter is placed in the superior vena cava; $\mathbf{B}$. The SL1 sheath slides over the catheter (which is stabilized in this position) until its tip comes into contact with the proximal poles of the ablation catheter resulting in an impedance change and shifting the part of the catheter shape out of the map; $\mathbf{C}$. Once the sheath slides a bit more distally, the distal poles of the ablation catheter come into contact with the sheath and the whole catheter shape jumps out from the map. This is a sign that the tip of the SL1 sheath is now in the place of the distal part of the catheter (position A). 


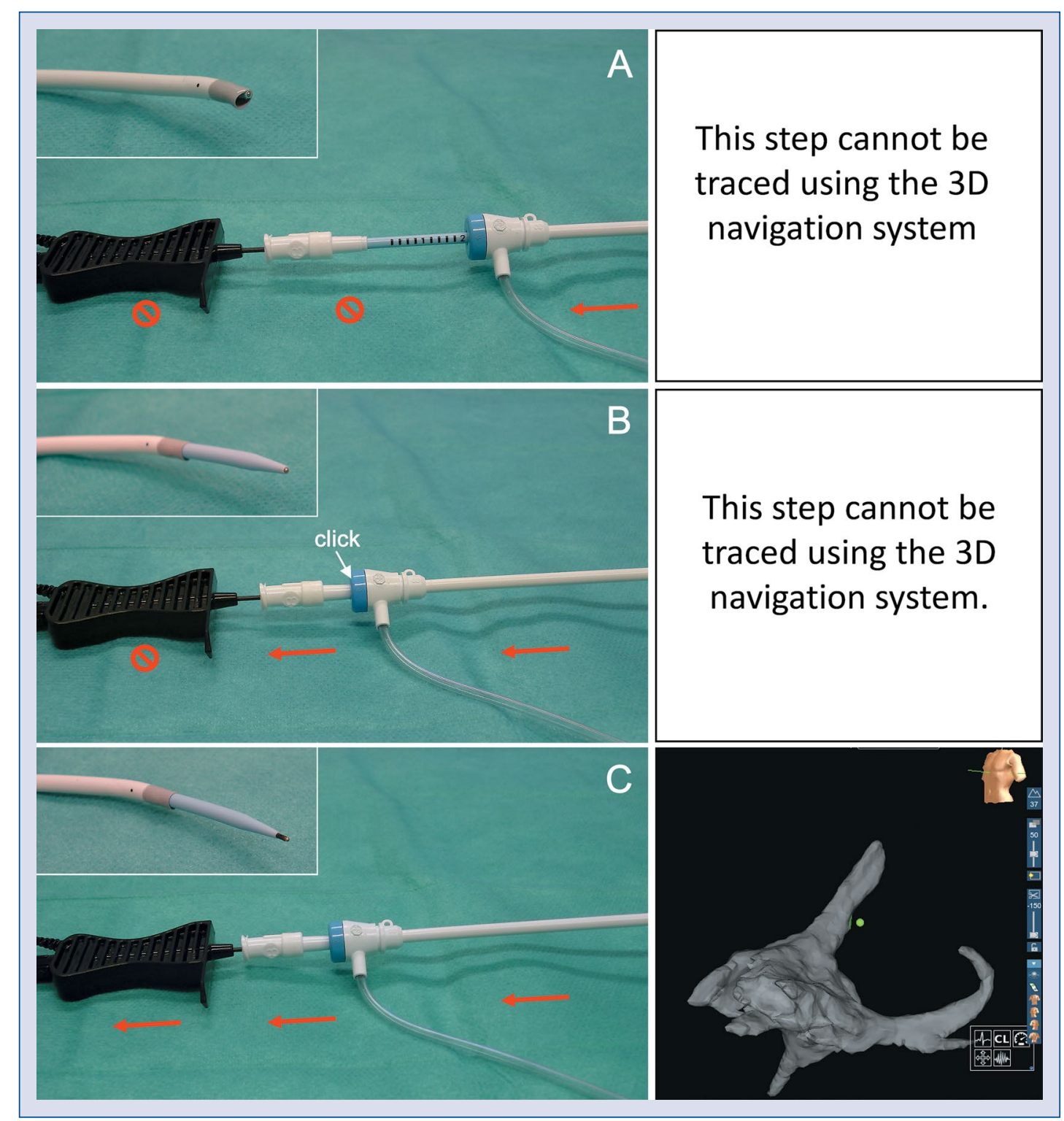

Figure 2. The steps of the "blind phase"; A. At the start position, the dilator is inserted into the sheath until the marker " 2 ". The radiofrequency (RF) needle is inserted into the dilator until the distance between the end of the pin on the needle and the dilator orifice is equal to the length of the pin itself. Now the needle and dilator are fixed and the sheath slides back over the dilator; B. The connection of the sheath with the dilator (a specific "click") confirms the connection. Now the dilator and the sheath are sliding back and the needle is still fixed; $\mathbf{C}$. The final position of the sheath dilator and the needle assembly before pulling back. The first two steps ( $A$ and $B$ ) cannot be traced using the three-dimensional (3D) navigation system. The right side (C) shows the 3D anatomical map of the right atrium, superior vena cava and coronary sinus in the left anterior oblique projection. The green point depicts the unipolar signal of the RF-needle once it appears out from the dilator. In the left upper corner of each picture, the corresponding picture of the distal part of the sheath is shown. Please note the gummy tip of the SL1 sheath, which offers additional safety.

position. After reaching the correct position, RF energy was delivered. Specific spontaneous echo contrasting occurred during the energy application on the TEE picture. The "tenting" of the IAS disappeared and the needle was inserted into the LA. The needle was aspirated and flushed with saline, and a spontaneous contrasting effect was seen at this moment in the LA, confirming the correct position of the needle (Fig. 3, Suppl. Videos 2, 3).

A single TSP was used and the ablation catheter was placed beside the multipolar mapping 


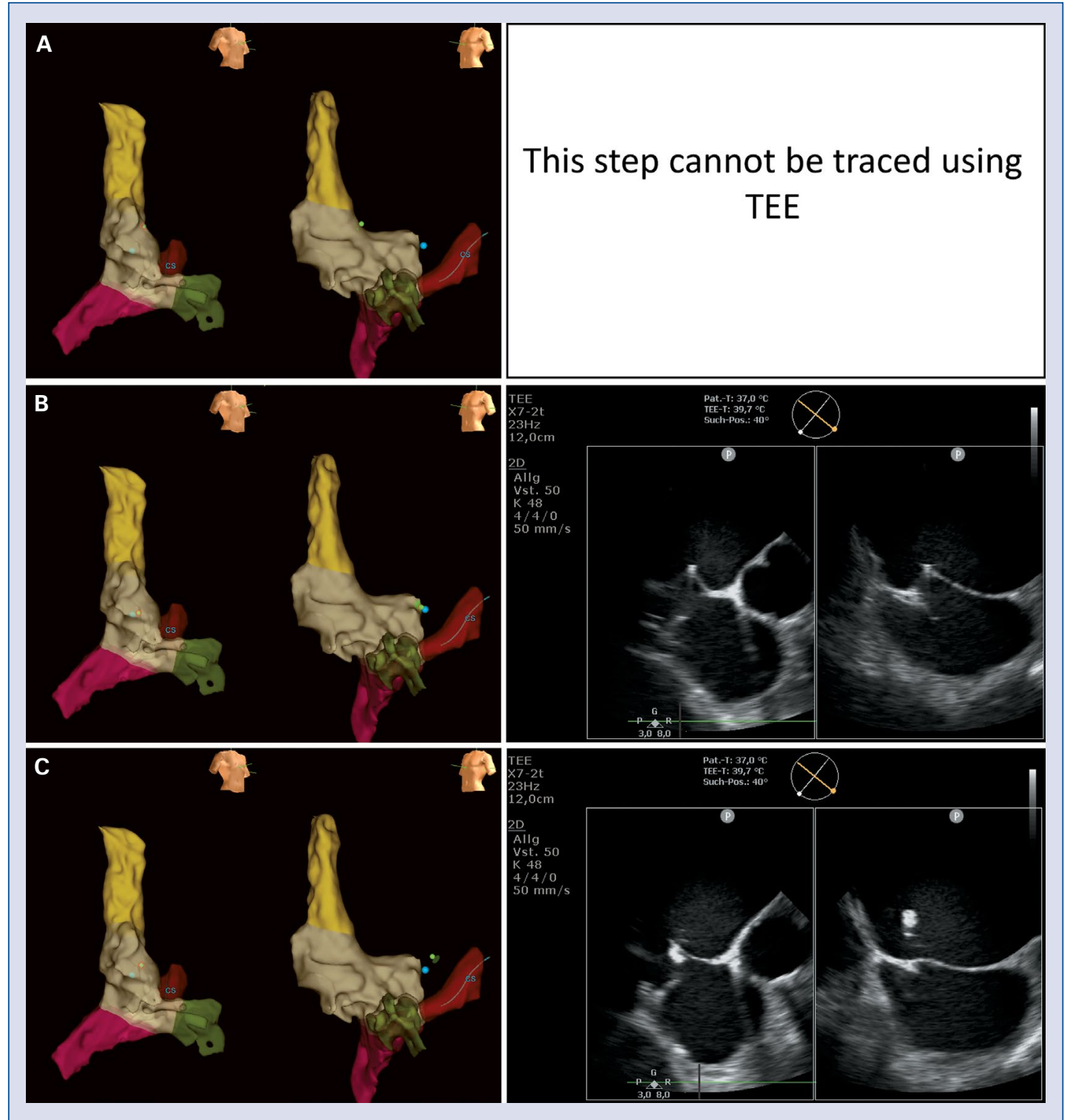

Figure 3. Left side three-dimensional anatomical map of the anatomical structures in the right anterior oblique and left anterior oblique projections; right atrium (grey), superior vena cava (SVC) (yellow), inferior vena cava (purple), coronary sinus (red) and right ventricle (green). The blue point depicts the ideal location for transseptal puncture, which was marked with the radiofrequency (RF)-catheter before. On the right side, transesophageal echocardiography (TEE) pictures of the interatrial septal (IAS) in short and bicaval planes; A. The RF-needle (the green point) moves from the SVC towards the IAS. This step cannot be traced using TEE; $\mathbf{B}$. The tip of the RF-needle reached the desired place. The TEE picture showing the "tenting" of the IAS confirms a safe position of the needle; $\mathbf{C}$. The tip of the needle is in the left atrium, out of the right atrium map. TEE pictures show specific spontaneous echo contrasting during energy application. Note, the "tenting" disappears after RF delivery.

catheter once the SL1 sheath was pulled back into the RA.

The mapping of LA was provided with a multipolar catheter (Advisor ${ }^{\mathrm{TM}}$ FL Circular Mapping Catheter, Sensor Enabled ${ }^{\mathrm{TM}}$, Abbott, USA, $\mathrm{n}=6$; Advisor $^{\mathrm{TM}}$ HD Grid Mapping Catheter, Sensor Enabled $^{\mathrm{TM}}$, Abbott, USA, $\mathrm{n}=4$ ).
At first the anatomy of all pulmonary veins were collected (LSPV-LIPV-RSPV-RIPV) followed by a complete geometry of the LA-box. Next the anatomy of the LA-roof was completed. The following step was mapping the left atrial appendage, the anterior wall and atrial septum. Finally, the inferior wall of the LA was mapped. 
Table 1. Periprocedural characteristics.

\begin{tabular}{|c|c|c|c|c|c|c|c|}
\hline Case & AF form & PVI & Re-PVI & $\begin{array}{c}\text { LAMRT } \\
\text { (linear ablation) }\end{array}$ & CFAEs & RAMRT & CTI \\
\hline 1 & Persistent & CRYO & RSPV, LIPV & - & - & - & - \\
\hline 2 & Persistent & CRYO & LSPV, RIPV & - & - & CT & Re-CTI \\
\hline 3 & Persistent & CRYO & - & $\begin{array}{l}\text { Left isthmus, } \\
\text { LA anterior }\end{array}$ & IAS, LA inferior & - & - \\
\hline 4 & Paroxysmal & CRYO & RSPV, RIPV & IAS & - & - & - \\
\hline 5 & Persistent & CRYO/RF & - & Left isthmus & - & - & - \\
\hline 6 & Persistent & CRYO/RF & RIPV & Left isthmus, IAS & $\begin{array}{l}\text { IAS, LA inferior, } \\
\text { LA posterior }\end{array}$ & - & - \\
\hline 7 & Persistent & CRYO & - & $\begin{array}{l}\text { Left isthmus, } \\
\text { LA-Roof, inferior } \\
\text { box-line }\end{array}$ & - & - & - \\
\hline 8 & Persistent & $\mathrm{RF}$ & All PVs & - & - & - & - \\
\hline 9 & Persistent & CRYO & RSPV & - & - & - & - \\
\hline 10 & Paroxysmal & CRYO & - & - & - & - & CTI \\
\hline
\end{tabular}

$\mathrm{AF}$ - atrial fibrillation; CFAE - complex fragmented atrial electrograms; CT — crista terminalis; CTI — cavo tricuspidal isthmus; IAS - inter atrial septum; LA - left atrium; LAMRT — left atrial macro re-entry tachycardia; LIPV — left inferior pulmonary vein; LSPV — left superior pulmonary vein; PVs - pulmonary veins; PVI - pulmonary vein isolation; RAMRT — right atrial macro re-entry tachycardia; RF — radiofrequency; RIPV — right inferior pulmonary vein; RSPV — right superior pulmonary vein

A voltage map was collected simultaneously with the anatomical map, which supplies additional information of the LA substrate. Pulmonary vein gaps were marked with tag points during mapping as well. During AF a complex fragmented atrial electrograms (CFAEs) map was also provided.

\section{Results}

Intravenous infusion of $49.5 \pm 27 \mathrm{~mL}$ Propofol $1 \%$ and $0.043 \pm 0,027 \mathrm{mg}$ Fentanyl injection was needed for stable sedation. No re-map was needed in this study.

After safe TSP was done in all cases, an LA anatomical and voltage map was created. The reisolation of pulmonary veins was the initial strategy. In 4 cases, all pulmonary veins were isolated. In 6 patients, re-PVI was performed ( 1 patient 4 PVs; 3 patients -2 PVs; 2 patients $-1 \mathrm{PV}$ ).

In 5 cases, linear ablation of the LA for treatment of left atrial macro re-entry tachycardia was provided. In 2 patients, focal atrial tachycardia was documented, 1 patient underwent cavo tricuspidal isthmus (CTI) ablation and 1 patient re-CTI ablation. The ablation of CFAEs was done in 2 patients. In 1 case, RA macro re-entry tachycardia was treated. Mean procedural time (the time from groin puncture until hemostasis, including waiting time) was $107.5 \pm 37.1 \mathrm{~min}$. No complications occurred during or after the procedure. Mean hospitalization time was $3 \pm 0.9$ days. All patients remained on anti-arrhythmic therapy for 3 months after ablation. After an 8.9 \pm 4 -month follow-up period, 2 patients had an arrhythmia recurrence. The periprocedural characteristics are presented in Table 1.

\section{Discussion}

\section{Non-fluoroscopy technique} for treatment of SVT

As mentioned above, a non-fluoroscopy approach using a 3D navigation system is increasingly recognized as a future technology in arrhythmia treatment. The treatment of right-sided SVT without fluoroscopy is already a well approved method and is a standard technique at many institutions including the one documented $[2,3]$. Herein, it is strongly recommended to start with right-sided SVT ablation once it has been decided to implement a non-fluoroscopy technique in your laboratory. Only after a learning curve is completed, consider starting with a non-fluoroscopy TSP [4].

\section{Non-fluoroscopy mapping and} ablation of the LA after TSP

To investigate the effectiveness and safety of the reconstruction of LA and PVI without fluoroscopy, Zhang et al. [5] enrolled 342 patients with paroxysmal AF. After X-ray-guided TSP, patients were divided in two groups - reconstruction of LA and PVI with and without the use of fluoroscopy. The X-ray time of LA reconstruction and PVI was 
$7.6 \pm 1.3 \mathrm{~min}$ in the group with fluoroscopy and the total X-ray exposure dose was more than 6-fold higher compared to the group without fluoroscopy. Fluoroscopy time before LA reconstruction was similar in both groups $(2.8 \pm 0.4$ and $2.4 \pm 0.6 \mathrm{~min})$ [5]. Recently, Raju et al. [6] published the experience using the near zero fluoroscopy ablation technique for complex LA ablations using the electroanatomic mapping system and TEE. The authors showed that the reduction of fluoroscopy time and radiation dose was statistically relevant in the nonfluoroscopy group compared with the control group. Moreover, in the majority of patients with patent foramen ovale (36\% in this study), zero fluoroscopy was possible [6].

The right-sided and left-sided atrial reconstruction without fluoroscopy using a 3D-navigation system seems to be an effective and safe method. The next most important question is; if the transseptal punction is also safe and effective when using a non-fluoroscopy technique. By rePVI, the incidence of the iatrogenic atrial septal defect after 12-36 months after PVI varies 20-37\% using cryoballoon and 9-19\% using the RFA technique. That means that up to one-third of re-PVI procedures after initial cryoballoon therapy (which have been increasing over the last few years) can be performed without using TSP [7].

\section{TSP without fluoroscopy}

Ferguson et al. [8] published a series documenting 21 patients with AF where 19 patients underwent TSP without fluoroscopy, using intracardiac echocardiography (ICE), and an electroanatomic mapping system. For double TSP, the authors used a conventional transseptal needle which was facilitated by electrocautery. This technique showed good results and was deemed safe in all cases [8]. Bulava et al. [9] demonstrated the result of ablation on 80 patients with paroxysmal $\mathrm{AF}$ randomized 1:1 ratio to undergo PVI with and without fluoroscopy. TSP in the group without fluoroscopy was performed by ICE guiding and using the CARTO 3 system. The total procedure and ablation time were comparable in both groups. All but 1 patient received zero fluoroscopy treatment in the non-fluoroscopy group. No procedure-related complications occurred in this study [9]. O'Brien et al. [10] demonstrated that after an adequate learning curve fluoroscopy-free TSP and ablation of complex left-sided atrial arrhythmias were safe and feasible in most patients. More recently, Sawhney et al. [11] published a stepwise approach to the non-fluoroscopy TSP technique in 32 patients us- ing an Endrys Coaxial needle, which was visualized on the CARTO system without the use of TEE or ICE. The TSP was performed safely and effectively using the non-fluoroscopic technique in a select group of patients [11].

\section{The RF transseptal needle}

Recently, a RF needle was developed. The first data showed that the use of an RF needle for TSP was associated with a lower total procedural time and lower risk of acute cerebral embolism during the catheter ablation of $\mathrm{AF}$ [12]. In this study Tokuda et al. [12] enrolled 383 consecutive patients who underwent catheter ablation for $\mathrm{AF}$ that required TSP using mechanical or RF transseptal needles. All patients had a cerebral magnetic resonance imaging performed 1 or 2 days after the procedure. Total procedure time was significantly shorter in the RF group than the non-RF group $(167 \pm 50$ vs. $181 \pm 52 \mathrm{~min}, \mathrm{p}=0.01)$. The incidence of acute cerebral embolism was lower in the $\mathrm{RF}$ group than the non-RF group (19 vs. $32 \%$, $\mathrm{p}=0.02)$. This can be attributed to some advantages of using the RF-needle: It enables crossing the thin aneurismal septum while reducing excessive tenting; can cross the fibrotic septum while reducing mechanical force; can cross the septum at a precise location; and the rounded atraumatic tip reduces the risk of skiving and embolism. As mentioned above, it also reduces transseptal procedure and fluoroscopy time vs. mechanical needle. Is easy to visualize on fluoroscopy (as well as on TEE) because of the radiopaque marker; and is easy to trace the tip of the needle on the 3D mapping system [13-16].

In the present study, the RF needle for TSP was used without the use of fluoroscopy. A single TSP was used and the ablation catheter was placed beside the multipolar mapping catheter. Double TSP can also be performed by repeating the abovedescribed steps.

According to available research, this is the first series of patients where the transseptal punction was performed with RF-needle using the non-fluoroscopy technique. With regard to the advantages described above, based on prior experience, it can be said that the RF needle is well suited for the non-fluoroscopy technique. Using this technique, it is possible to:

- Trace the RF-needle tip in the 3D navigation system before, during and after TSP (minimizing the blind phase);

- To see the highly echogenic tip of the RF-needle in the TEE image; 
- To monitor the pressure shift during the TSP;

- To see the spontaneous contrast effect in the LA while injecting the saline in the RF-needle after TSP.

This method is not only effective, but also safe. The safety concern in the present study was based on the following points:

- Always sliding the sheath, introducer and the needle backwards during the blind phase;

- Live TEE imaging during the TSP;

- Adequate sedation of the patient, which allows a stable map; the soft tip of the SL1 sheath provides better safety.

\section{Limitations of the study}

The non-fluoroscopy group of patients was not compared with conventional groups. Limited number of patients.

\section{Conclusions}

Non-fluoroscopy TSP using an RF needle, traced with the 3D navigation system and TEE is a safe and effective technique. Further investigation of this method is needed.

Conflict of interest: Philipp Sommer - Consultant for Abbott, Boston Scientific, BiosenseWebster and Medtronic.

\section{References}

1. Gaita F, Guerra PG, Battaglia A, et al. The dream of near-zero X-rays ablation comes true. Eur Heart J. 2016; 37(36): 2749-2755, doi: 10.1093/eurheartj/ehw223, indexed in Pubmed: 27354053.

2. Álvarez M, Bertomeu-González V, Arcocha MFe, et al. investigators of the Spanish Multicenter Registry of Fluoroscopyfree Ablation. Nonfluoroscopic Catheter Ablation. Results From a Prospective Multicenter Registry. Rev Esp Cardiol (Engl Ed). 2017; 70(9): 699-705, doi: 10.1016/j.rec.2016.12.040, indexed in Pubmed: 28159569

3. Seizer P, Bucher V, Frische C, et al. Efficacy and safety of zerofluoroscopy ablation for supraventricular tachycardias. Use of optional contact force measurement for zero-fluoroscopy ablation in a clinical routine setting. Herz. 2016; 41(3): 241-245, doi: 10.1007/s00059-015-4358-4, indexed in Pubmed: 26462477.

4. Gist K, Tigges C, Smith G, et al. Learning curve for zerofluoroscopy catheter ablation of AVNRT: early versus late experience. Pacing Clin Electrophysiol. 2011; 34(3): 264-268, doi: 10.1111/j.1540-8159.2010.02952.x, indexed in Pubmed: 21070259.

5. Zhang JQ, Yu RH, Liang JB, et al. Reconstruction left atrium and isolation pulmonary veins of paroxysmal atrial fibrillation using single contact force catheter with zero x-ray exposure: a CONSORT Study. Medicine (Baltimore). 2017; 96(41): e7726, doi: 10.1097/MD.0000000000007726, indexed in Pubmed: 29019873.

6. Raju H, Whitaker J, Taylor C, et al. Electroanatomic Mapping and Transoesophageal Echocardiography for near Zero Fluoroscopy during Complex Left Atrial Ablation. Heart Lung Circ. 2016; 25(7): 652-660, doi: 10.1016/j.hlc.2016.01.018, indexed in Pubmed: 26979468.

7. Naksuk N, Asirvatham SJ, Naksuk N, et al. Iatrogenic atrial septal defect: reassurance or inquisitiveness. J Interv Card Electrophysiol. 2018; 52(2): 137-140, doi: 10.1007/s10840-018-0369-4, indexed in Pubmed: 29680973.

8. Ferguson JD, Helms A, Mangrum JM, et al. Catheter ablation of atrial fibrillation without fluoroscopy using intracardiac echocardiography and electroanatomic mapping. Circ Arrhythm Electrophysiol. 2009; 2(6): 611-619, doi: 10.1161/CIRCEP.109.872093, indexed in Pubmed: 20009075.

9. Bulava A, Hanis J, Eisenberger M. Catheter ablation of atrial fibrillation using zero-fluoroscopy technique: a randomized trial. Pacing Clin Electrophysiol. 2015; 38(7): 797-806, doi: 10.1111/ pace.12634, indexed in Pubmed: 25790320.

10. O'Brien B, Balmforth DC, Hunter RJ, et al. Fluoroscopy-free AF ablation using transesophageal echocardiography and electroanatomical mapping technology. J Interv Card Electrophysiol. 2017; 50(3): 235-244, doi: 10.1007/s10840-017-0288-9, indexed in Pubmed: 29134434.

11. Sawhney V, Breitenstein A, Watts T, et al. A novel technique for performing transseptal puncture guided by a non-fluoroscopic 3D mapping system. Pacing Clin Electrophysiol. 2019; 42(1): 4-12, doi: 10.1111/pace.13541, indexed in Pubmed: 30397922.

12. Tokuda M, Yamashita S, Matsuo S, et al. Radiofrequency needle for transseptal puncture is associated with lower incidence of thromboembolism during catheter ablation of atrial fibrillation: propensity score-matched analysis. Heart Vessels. 2018; 33(10): 1238-1244, doi: 10.1007/s00380-018-1159-8, indexed in Pubmed: 29637262.

13. Fromentin S, Sarrazin JF, Champagne J, et al. Prospective comparison between conventional transseptal puncture and transseptal needle puncture with radiofrequency energy. J Interv Card Electrophysiol. 2011; 31(3): 237-242, doi: 10.1007/s10840-0119564-2, indexed in Pubmed: 21503731.

14. Smelley MP, Shah DP, Weisberg I, et al. Initial experience using a radiofrequency powered transseptal needle. J Cardiovasc Electrophysiol. 2010; 21(4): 423-427, doi: 10.1111/j.15408167.2009.01656.x, indexed in Pubmed: 19925604.

15. Winkle RA, Mead RH, Engel G, et al. The use of a radiofrequency needle improves the safety and efficacy of transseptal puncture for atrial fibrillation ablation. Heart Rhythm. 2011; 8(9): 1411-1415, doi: 10.1016/j.hrthm.2011.04.032, indexed in Pubmed: 21699841.

16. Feld GK, Tiongson J, Oshodi G. Particle formation and risk of embolization during transseptal catheterization: comparison of standard transseptal needles and a new radiofrequency transseptal needle. J Interv Card Electrophysiol. 2011; 30(1): 31-36, doi: 10.1007/s10840-010-9531-3, indexed in Pubmed: 21249439. 